\title{
Optimal Designs for Stated Choice Experiments that Incorporate Ties
}

\author{
Stephen Bush*, Leonie Burgess*, Deborah Street* \\ Department of Mathematical Sciences, University of Technology Sydney, PO Box 123 \\ Broadway NSW 2007, Australia
}

\begin{abstract}
In 1970 Davidson generalised the Bradley-Terry model to allow respondents to say that the two options presented in a choice task were equally attractive. In this paper we extend this idea to the MNL model with $m$ options in each choice set and we show that the optimal designs for the MNL model are also optimal in this setting.
\end{abstract}

Keywords: Paired comparisons, Multiple comparisons, Bradley-Terry model, Multinomial logit model, Davidson ties model

\section{Introduction}

Stated choice experiments are a common method for modelling decision making behaviour. These experiments are used in many disciplines, including marketing, health economics, tourism, and public policy. Louviere et al. (2000) and Train (2002) provide a good introduction to the area.

We begin with a small example based on Gerard, Sailsbury, Street, Pope and Baxter (2008). These authors were interested in determining "the relative importance of factors that influence patient choice in the booking of general practice appointments for two health problems'. They identified four attributes that might be important. They are given in Table 1. Using these levels we can describe 48 appointments (profiles, items, or treatment combinations). One such appointment is (5 days later, a doctor of my choice, at a time convenient to me, for about $10 \mathrm{mins}$ ). We use these profiles to

\footnotetext{
${ }^{*}$ Corresponding author. Tel.: +61-2-9514-2246

Email address: stephen.bush@uts.edu.au (Stephen Bush)
} 


\begin{tabular}{|c|c|c|c|c|}
\hline Attribute & Level 1 & Level 2 & Level 3 & Level 4 \\
\hline $\begin{array}{l}\text { Day of } \\
\text { appointment }\end{array}$ & Same day & Next day & $\begin{array}{l}5 \text { days } \\
\text { later }\end{array}$ & $\begin{array}{c}10 \text { days } \\
\text { later }\end{array}$ \\
\hline $\begin{array}{l}\text { Professional } \\
\text { person }\end{array}$ & Nurse & $\begin{array}{l}\text { Doctor, any } \\
\text { available }\end{array}$ & $\begin{array}{l}\text { Doctor of } \\
\text { choice }\end{array}$ & \\
\hline $\begin{array}{c}\text { Time of day of } \\
\text { appointment }\end{array}$ & Inconvenient & Convenient & & \\
\hline $\begin{array}{l}\text { Length of } \\
\text { appointment }\end{array}$ & 10 mins & 20 mins & & \\
\hline
\end{tabular}

Table 1: Attributes and levels for the GP appointment experiment

construct choice sets with, say, three options, and ask each respondent to choose one of the options. A possible choice set is given in Table 2. Each

\begin{tabular}{lll}
\hline Appointment A & Appointment B & Appointment $\mathbf{C}$ \\
\hline Same Day & 5 days later & 10 days later \\
Nurse & Any available Doctor & Doctor of choice \\
Convenient & Convenient & Inconvenient \\
20 mins & 10 mins & 20 mins \\
\hline
\end{tabular}

Table 2: Example of a choice set

respondent is shown all of the choice sets in the experiment, and for each choice set they are asked to choose one of the options presented. Provided that the choice experiment has been correctly designed, these responses can be used to estimate the effects of each of the attributes on the booking of general practice appointments, and to estimate the effects of the interactions of any two of the attributes on the booking of general practice appointments.

In general we will describe the items to be compared by $k$ attributes, where we assume that the $q^{\text {th }}$ attribute has $\ell_{q}$ levels, represented by $0,1, \ldots, \ell_{q}-$ 1 , and that all choice sets in an experiment have $m$ options. We assume that no choice set contains a repeated option and that no choice set is repeated in an experiment. We also assume that the options are generic and unlabelled.

In these circumstances, if respondents are forced to choose one of the 
items in each choice set, a number of results about the optimal design exist; see Burgess and Street (2003, 2005), as well as Street and Burgess (2007) who summarise this and related work. This work assumed the multinomial logit (MNL) model. In choice sets of size 2 the MNL model coincides with the Bradley-Terry model.

Sometimes, of course, respondents are unable to express a preference for only one of the items in the choice set. For example a respondent may find Appointments B and C from Table 2 equally attractive. Davidson (1970) extended the Bradley-Terry model so that ties could be accommodated in a paired comparison experiment.

The aim of this paper is to extend Davidson's work to allow for an arbitrary number of options in each choice set. We establish that designs that are optimal in an MNL setting are also optimal for the estimation of the corresponding effects when using the generalised Davidson ties model.

\section{Davidson Ties Model}

One model that is used to analyse stated choice experiments is the BradleyTerry paired comparison model, or simply the Bradley-Terry model. In this model, we estimate the relative merit of each item using the preferences collected. We denote the merit of an item $T_{i}$ as $\pi_{i}$, and impose the normalising constraint $\prod_{i}^{t} \pi_{i}=1$. We use these merits to find the probability of selecting a particular item $T_{i}$ when compared to another item $T_{j}$. When item $T_{i}$ appears with item $T_{j}$ in a choice set, then the probability that item $T_{i}$ is selected from the choice set is

$$
P\left(T_{i} \mid\left\{T_{i}, T_{j}\right\}\right)=\frac{\pi_{i}}{\pi_{i}+\pi_{j}} .
$$

Davidson (1970) gives a modification of the Bradley-Terry model that incorporates ties. He suggests that the probability of a tie between items should be proportional to the geometric mean of the merits of these items. Thus the merit of the set $\left\{T_{i}, T_{j}\right\}$ is given by $\pi_{\{i, j\}}=\nu \sqrt{\pi_{i} \pi_{j}}$. As a consequence, when the merits of the items are similar, the probability of a respondent stating that they cannot choose between the items will be greater. That is, assuming that $\nu$ is independent of items $T_{i}$ and $T_{j}$, the preference probabilities with ties incorporated into the Bradley-Terry model are (from Davidson (1970))

$$
P\left(T_{i} \mid\left\{T_{i}, T_{j}\right\}\right)=\frac{\pi_{i}}{\pi_{i}+\pi_{j}+\nu \sqrt{\pi_{i} \pi_{j}}},
$$




$$
\begin{aligned}
P\left(T_{j} \mid\left\{T_{i}, T_{j}\right\}\right) & =\frac{\pi_{j}}{\pi_{i}+\pi_{j}+\nu \sqrt{\pi_{i} \pi_{j}}} \text {, and } \\
P\left(\left\{T_{i}, T_{j}\right\} \mid\left\{T_{i}, T_{j}\right\}\right) & =\frac{\nu \sqrt{\pi_{i} \pi_{j}}}{\pi_{i}+\pi_{j}+\nu \sqrt{\pi_{i} \pi_{j}}} .
\end{aligned}
$$

\section{Optimal Designs for the Davidson Ties Model}

In this section we prove results that give optimal designs when the Davidson ties model is used. We begin by constructing the information matrix for the estimation of attribute effects and the ties parameter $\nu$. We use this expression to prove that the optimal designs for the Bradley-Terry model are also optimal for the Davidson ties model.

We let $\Lambda(\boldsymbol{\pi}, \nu)$ be the matrix of second derivatives of the likelihood function, where $\boldsymbol{\pi}=\left(\pi_{1}, \pi_{2}, \ldots, \pi_{t}\right)$, where $t$ is the number of possible items. We can partition $\Lambda(\boldsymbol{\pi}, \nu)$ into four blocks

$$
\Lambda(\boldsymbol{\pi}, \nu)=\left[\begin{array}{ll}
\Lambda_{\gamma \gamma}(\boldsymbol{\pi}, \nu) & \Lambda_{\nu \gamma}(\boldsymbol{\pi}, \nu) \\
\Lambda_{\gamma \nu}(\boldsymbol{\pi}, \nu) & \Lambda_{\nu \nu}(\boldsymbol{\pi}, \nu)
\end{array}\right]
$$

$\Lambda_{\gamma \gamma}(\boldsymbol{\pi}, \nu)$ is a $t \times t$ matrix containing the second derivatives with respect to two of the entries in $\gamma=\ln (\boldsymbol{\pi}) . \Lambda_{\gamma \nu}(\boldsymbol{\pi}, \nu)$ is a $t \times 1$ vector that contains the second derivatives with respect to one entry in $\gamma$ and $\nu$, and $\Lambda_{\nu \gamma}(\boldsymbol{\pi}, \nu)=I_{\gamma \nu}(\boldsymbol{\pi}, \nu)^{T}$. $\Lambda_{\nu \nu}(\boldsymbol{\pi}, \nu)$ contains the second derivative with respect to $\nu$.

If we make the assumption of equal merits then the expressions for the entries of $\Lambda$ are simple. We will leave $\nu$ unspecified as was done in Davidson (1970). We are then making the assumption that designs which are efficient when the merits of the items are equal will also be efficient for other values of $\boldsymbol{\pi}$. For small cases that we have investigated this has proven to be a reasonable assumption; see Street and Burgess (2007) and Street and Burgess (2004). That is, we assume $\boldsymbol{\pi}=\mathbf{j}=\boldsymbol{\pi}_{0}$, to obtain

$$
\begin{aligned}
\Lambda_{\gamma \gamma}\left(\boldsymbol{\pi}_{0}, \nu\right)_{i i} & =\frac{1}{2 N(2+\nu)} \sum_{C \mid T_{i} \in C} n_{C}, \\
\Lambda_{\gamma \gamma}\left(\boldsymbol{\pi}_{0}, \nu\right)_{i j} & =\frac{-1}{2 N(2+\nu)} \sum_{C \mid T_{i}, T_{j} \in C} n_{C}, \\
\Lambda_{\gamma \nu}\left(\boldsymbol{\pi}_{0}, \nu\right)_{1 i} & =0, \text { and } \\
\Lambda_{\nu \nu}\left(\boldsymbol{\pi}_{0}, \nu\right) & =\frac{2}{\nu(2+\nu)^{2}}
\end{aligned}
$$


where $n_{C}$ equals 1 if the choice set $C$ appears in the experiment and 0 if $C$ does not appear in the experiment.

As in Street and Burgess (2007), we are interested in estimating the contrasts $B_{\gamma} \gamma$ as well as $\nu$. These contrasts whose coefficients are in $B_{\gamma}$ may correspond to the main effects of the attributes, or two-factor interactions between attributes, or perhaps subsets of these. We are not interested in estimating contrasts that include both $\nu$ and entries in $\gamma$, but we do want to estimate $\nu$ itself. We can construct a matrix $B$ that contains both the contrast matrix $B_{\gamma}$ and the effect of $\nu$.

$$
B=\left[\begin{array}{cc}
B_{\gamma} & \mathbf{0} \\
\mathbf{0} & B_{\nu}
\end{array}\right]=\left[\begin{array}{cc}
B_{\gamma} & \mathbf{0} \\
\mathbf{0} & 1
\end{array}\right] .
$$

Then the information matrix for estimating the contrasts in $B_{\gamma}$ and the ties parameter $\nu$ is

$$
C\left(\boldsymbol{\pi}_{0}, \nu\right)=B \Lambda\left(\boldsymbol{\pi}_{0}, \nu\right) B^{T}=\left[\begin{array}{cc}
B_{\gamma} \Lambda_{\gamma \gamma}\left(\boldsymbol{\pi}_{0}, \nu\right) B_{\gamma}^{T} & \mathbf{0} \\
\mathbf{0} & \frac{2}{\nu(2+\nu)^{2}}
\end{array}\right] .
$$

Suppose that we assume $\boldsymbol{\pi}=\boldsymbol{\pi}_{0}$. Then the information matrix for the estimation of the entries in $\gamma$ when the Bradley-Terry model is used is denoted $\Lambda\left(\boldsymbol{\pi}_{0}\right)_{\mathrm{B}-\mathrm{T}}$. The form of this matrix is given in El-Helbawy et al. (1994). Also suppose that $\Lambda_{\gamma \gamma}\left(\boldsymbol{\pi}_{0}, \nu\right)_{\mathrm{DAV}}$ is the $(1,1)$ block of the information matrix for the estimation of the entries in $\gamma$ plus $\nu$ when the Davidson ties model is used. Then if we compare the diagonal entries of both matrices, we see that

$$
2(2+\nu)\left(\Lambda_{\gamma \gamma}\left(\boldsymbol{\pi}_{0}, \nu\right)_{\mathrm{DAV}}\right)_{i i}=4\left(\Lambda\left(\boldsymbol{\pi}_{0}\right)_{\mathrm{B}-\mathrm{T}}\right)_{i i}=\frac{1}{N} \sum_{C \mid T_{i} \in C} n_{C} .
$$

If we compare the off-diagonal entries in the two matrices, we see that

$$
2(2+\nu)\left(\Lambda_{\gamma \gamma}\left(\boldsymbol{\pi}_{0}, \nu\right)_{\mathrm{DAV}}\right)_{i j}=4\left(\Lambda\left(\boldsymbol{\pi}_{0}\right)_{\mathrm{B}-\mathrm{T}}\right)_{i j}=\frac{1}{N} \sum_{C \mid T_{i}, T_{j} \in C} n_{C} .
$$

Thus we have

$$
\Lambda\left(\boldsymbol{\pi}_{0}, \nu\right)_{\mathrm{DAV}}=\left[\begin{array}{cc}
\frac{2}{2+\nu} \Lambda\left(\boldsymbol{\pi}_{0}\right)_{\mathrm{B}-\mathrm{T}} & \mathbf{0} \\
\mathbf{0} & \frac{2}{\nu(2+\nu)^{2}}
\end{array}\right] .
$$

Since we can express these information matrices in terms of each other, we may now look at comparing optimality results for these two models. We will use the $D$-optimality criterion. 
Theorem 1. For a set of $p$ contrasts of the entries in $\gamma$ and a constant but unknown $\nu$, the D-optimal design for the estimation of the contrasts of $\gamma$ over a set of competing designs $\mathfrak{X}$ when the Bradley-Terry model is used will also be D-optimal for the estimation of the same contrasts and $\nu$ over the same set of competing designs when estimating the Davidson ties model.

Proof. We begin by letting $B$ be the matrix of Equation 1 . Then the information matrix for the estimation of $B_{\gamma} \gamma$ plus $\nu$ when the Davidson ties model is used, $C\left(\boldsymbol{\pi}_{0}, \nu\right)_{\mathrm{DAV}}$, is

$$
C\left(\boldsymbol{\pi}_{0}, \nu\right)_{\mathrm{DAV}}=\left[\begin{array}{cc}
B_{\gamma} \Lambda_{\gamma \gamma}\left(\boldsymbol{\pi}_{0}, \nu\right)_{\mathrm{DAV}} B_{\gamma}^{T} & \mathbf{0} \\
\mathbf{0} & \frac{2}{\nu(2+\nu)^{2}}
\end{array}\right] .
$$

Then using Equation 2, and simplifying, we obtain

$$
C\left(\boldsymbol{\pi}_{0}, \nu\right)_{\mathrm{DAV}}=\left[\begin{array}{cc}
\frac{2}{2+\nu} C\left(\boldsymbol{\pi}_{0}\right)_{\mathrm{B}-\mathrm{T}} & \mathbf{0} \\
\mathbf{0} & \frac{2}{\nu(2+\nu)^{2}}
\end{array}\right] .
$$

Thus we see that

$$
\operatorname{det}\left(C\left(\boldsymbol{\pi}_{0}, \nu\right)_{D A V}\right)=\frac{2^{p+1}}{\nu(2+\nu)^{p+2}} \operatorname{det}\left(C\left(\boldsymbol{\pi}_{0}\right)_{\mathrm{B}-\mathrm{T}}\right) .
$$

Since

$$
\operatorname{det}\left(\left(C\left(\boldsymbol{\pi}_{0}\right)_{\mathrm{B}-\mathrm{T}}\right)_{\xi_{\mathrm{OPT}}}\right) \geq \operatorname{det}\left(\left(C\left(\boldsymbol{\pi}_{0}\right)_{\mathrm{B}-\mathrm{T}}\right)_{\xi}\right)
$$

for all $\xi \in \mathfrak{X}$, the relative efficiency of a generic design $\xi$ compared to $\xi_{\mathrm{OPT}}$, the design that is optimal for estimating $B_{\gamma} \gamma$ when the Bradley-Terry model is used, when the Davidson ties model is used is

$$
\begin{aligned}
D_{\mathrm{eff}}\left(\xi, \xi_{\mathrm{OPT}}\right) & =\left(\frac{\frac{2^{p+1}}{\nu(2+\nu)^{p+2}} \operatorname{det}\left(\left(C\left(\boldsymbol{\pi}_{0}\right)_{\mathrm{B}-\mathrm{T}}\right)_{\xi}\right)}{\frac{2^{p+1}}{\nu(2+\nu)^{p+2}} \operatorname{det}\left(\left(C\left(\boldsymbol{\pi}_{0}\right)_{\mathrm{B}-\mathrm{T}}\right)_{\xi_{\mathrm{OPT}}}\right)}\right)^{\frac{1}{p+1}} \\
& =\left(\frac{\operatorname{det}\left(\left(C\left(\boldsymbol{\pi}_{0}\right)_{\mathrm{B}-\mathrm{T}}\right)_{\xi}\right)}{\operatorname{det}\left(\left(C\left(\boldsymbol{\pi}_{0}\right)_{\mathrm{B}-\mathrm{T}}\right)_{\xi_{\mathrm{OPT}}}\right)}\right)^{\frac{1}{p+1}} \\
& \leq 1
\end{aligned}
$$

for all $\xi \in \mathfrak{X}$. Therefore, by the definition of $D$-optimality, $\xi_{\mathrm{OPT}}$ is the $D$ optimal design for the estimation of the contrasts in $B_{\gamma}$ plus $\nu$ when the Davidson ties model is used. 


\section{The Generalised Davidson Ties Model}

Luce (1959) extends the Bradley-Terry model to accommodate the comparison of more than two items. Suppose that we present $m$ items $\left\{T_{i_{1}}, \ldots, T_{i_{m}}\right\}=$ $C$ to the respondent. Then we can estimate a merit, $\pi_{i}$, for each of these items. As in the Bradley-Terry model, we let $V_{i}=\ln \left(\pi_{i}\right)$ be the deterministic part of the utility function. Then the probability that the item $T_{i} \in C$ is chosen is

$$
P\left(T_{i} \mid C\right)=\frac{\pi_{i}}{\sum_{a=1}^{m} \pi_{i_{a}}}
$$

We call this model the multinomial logit model (MNL model).

In this section we consider a generalisation of the MNL model so that it accommodates ties. This generalisation is analogous to Davidson's generalisation of the Bradley-Terry model. We will first set up the model and derive the information matrix for the estimation of a set of contrasts and the ties parameter.

We begin this section by returning to the experiment introduced in Section 1, looking at how we can allow for ties when we have larger choice sets.

Example 1. Consider the experiment introduced in Section 1. Suppose that we create choice sets with three items each. One such choice set is $\{00,01,10\}$. Then if we allow respondents to state that a subset of these items are equally attractive then there are 7 different outcomes that could arise from the choice set. The respondent could state a preference for a single item, 00,01 or 10 , state that a pair of items are equally attractive, $\{00,01\},\{00,10\}$ or $\{01,10\}$, or state that all three items are equally attractive, $\{00,01,10\}$.

Here we notice that the respondent is not only permitted to find pairs of items in the choice set equally attractive, but is also permitted to state that larger subsets of the items in the choice set are equally attractive. In his paper, Davidson argues that the merit of finding a set of items equally attractive is proportional to the geometric mean of the item merits. We will assume, as Davidson did, that the proportionality is constant across choice sets is strictly positive. If this constant is equal to zero then this means that no respondent has stated that any of the items in any of the choice sets are equally attractive, and in this case the MNL model should be used instead.

Example 2. Consider the choice set introduced in Example 1. If we assign merits $\pi_{00}, \pi_{01}$, and $\pi_{10}$ to the items in the choice set then we obtain merits 
$\nu \sqrt{\pi_{00} \pi_{01}}, \nu \sqrt{\pi_{00} \pi_{10}}$ and $\nu \sqrt{\pi_{01} \pi_{10}}$ for ties between pairs of items, and the merit of the set of size 3 is $\nu \sqrt[3]{\pi_{00} \pi_{01} \pi_{10}}$. Let

$D_{\{00,01,10\}}=\pi_{00}+\pi_{01}+\pi_{10}+\nu \sqrt{\pi_{00} \pi_{01}}+\nu \sqrt{\pi_{00} \pi_{10}}+\nu \sqrt{\pi_{01} \pi_{10}}+\nu \sqrt[3]{\pi_{00} \pi_{01} \pi_{10}}$.

Then the selection probabilities of each of the types of choices take the form

$$
\begin{aligned}
P(00 \mid\{00,01,10\}) & =\frac{\pi_{00}}{D_{\{00,01,10\}}}, \\
P(\{00,01\} \mid\{00,01,10\}) & =\frac{\nu \sqrt{\pi_{00} \pi_{01}}}{D_{\{00,01,10\}}}, \\
& \vdots \\
P(\{00,01,10\} \mid\{00,01,10\}) & =\frac{\nu \sqrt[3]{\pi_{00} \pi_{01} \pi_{10}}}{D_{\{00,01,10\}}} .
\end{aligned}
$$

In general, let the merit of item $T_{i}$ be $\pi_{i}$, the merit of the set of items $T_{i_{1}}$ and $T_{i_{2}}$ be $\nu \sqrt{\pi_{i_{1}} \pi_{i_{2}}}$. Let the merit of the set of items $T_{i_{1}}, T_{i_{2}}$ and $T_{i_{3}}$ be $\nu \sqrt[3]{\pi_{i_{1}} \pi_{i_{2}} \pi_{i_{3}}}$. We continue this until we get to the respondent finding all of the $m$ items in the choice set equally attractive, with corresponding merit $\nu \sqrt[m]{\pi_{i_{1}} \pi_{i_{2}} \ldots \pi_{i_{m}}}$. As in the MNL model, to obtain the probability of making a particular decision, we divide the merit of that decision by the sum of the merits of all possible decisions from the choice set. For a choice set $C=\left\{T_{i_{1}}, T_{i_{2}}, \ldots, T_{i_{m}}\right\}$, we denote the sum of the merits for each possible decision as $D_{C}$. That is,

$$
D_{C}=\sum_{a=1}^{m} \pi_{i_{a}}+\sum_{x=2}^{m} \sum_{\left\{T_{j_{1}}, \ldots, T_{j_{x}}\right\} \subseteq C} \nu \sqrt[x]{\pi_{j_{1}} \ldots \pi_{j_{x}}} .
$$

We can then express the probabilities for each decision as

$$
\begin{aligned}
P\left(T_{i_{1}} \mid C\right) & =\frac{\pi_{i_{1}}}{D_{C}} \\
P\left(\left\{T_{i_{1}}, T_{i_{2}}\right\} \mid C\right) & =\frac{\nu \sqrt{\pi_{i_{1}} \pi_{i_{2}}}}{D_{C}}, \\
& \vdots \\
P\left(\left\{T_{i_{1}}, T_{i_{2}}, \ldots, T_{i_{m}}\right\} \mid C\right) & =\frac{\nu \sqrt[m]{\pi_{i_{1}} \pi_{i_{2}} \ldots \pi_{i_{m}}}}{D_{C}} .
\end{aligned}
$$


If we assume, as did Davidson (1970), the null hypothesis of equal merits for each of the items and that $\nu$ is left unspecified, then the expression for $\Lambda(\boldsymbol{\pi}, \nu)$ can be simplified; that is we assume that $\boldsymbol{\pi}=\boldsymbol{j}=\boldsymbol{\pi}_{0}$. Then using this we have

$$
\begin{aligned}
\Lambda_{\gamma \gamma}\left(\boldsymbol{\pi}_{0}, \nu\right)_{i j} & =\frac{\nu \sum_{x=2}^{m}\left(\left(\frac{(x-1)}{x m(m-1)}-\frac{1}{m^{2}}\right)\left(\begin{array}{c}
m \\
x
\end{array}\right)\right)-\frac{1}{m}}{N\left(m+\nu \sum_{x=2}^{m}\left(\begin{array}{c}
m \\
x
\end{array}\right)\right)} \sum_{C \mid T_{i}, T_{j} \in C} n_{C}, \\
\Lambda_{\gamma \gamma}\left(\boldsymbol{\pi}_{0}, \nu\right)_{i i} & =\frac{1-\frac{1}{m}+\nu \sum_{x=2}^{m}\left(\frac{1}{m x}-\frac{1}{m^{2}}\right)\left(\begin{array}{c}
m \\
x
\end{array}\right)}{N\left(m+\nu \sum_{x=2}^{m}\left(\begin{array}{c}
m \\
x
\end{array}\right)\right)} \sum_{C \mid T_{i} \in C} n_{C}, \\
\Lambda_{\gamma \nu}\left(\boldsymbol{\pi}_{0}, \nu\right)_{i} & =0, \text { and } \\
\Lambda_{\nu \nu}\left(\boldsymbol{\pi}_{0}, \nu\right) & =\frac{m \sum_{x=2}^{m}\left(\begin{array}{c}
m \\
x
\end{array}\right)}{\nu\left(m+\nu \sum_{x=2}^{m}\left(\begin{array}{c}
m \\
x
\end{array}\right)\right)^{2}} .
\end{aligned}
$$

$\Lambda_{\nu \nu}\left(\pi_{0}, \nu\right)$ is a function of $m$ and $\nu$ only and we hence refer to this entry as $\Lambda_{\nu \nu}(m, \nu)$.

\section{Optimal Designs for the Generalised Davidson Ties Model}

In this section we prove results that give optimal designs when the generalised Davidson ties model is used. Again we show that the optimal designs for the MNL model are optimal for the generalised Davidson ties model for the corresponding effects.

The information matrix for estimating $\nu$ and the contrasts in $B_{\gamma} \gamma$, under the null hypothesis of equal merits, is

$$
C\left(\boldsymbol{\pi}_{0}, \nu\right)=\left[\begin{array}{cc}
B_{\gamma} \Lambda_{\gamma \gamma}\left(\boldsymbol{\pi}_{0}, \nu\right) B_{\gamma}^{T} & \mathbf{0} \\
\mathbf{0} & \Lambda_{\nu \nu}(m, \nu)
\end{array}\right]
$$

If we compare the form for $\Lambda\left(\pi_{0}\right)_{\mathrm{MNL}}$, the information matrix for the estimation of $\gamma=\ln (\boldsymbol{\pi})$ when the MNL model is used as given in Burgess and Street (2005), to the first block of $\Lambda\left(\boldsymbol{\pi}_{0}, \nu\right)$ when the generalised Davidson ties model is used, denoted here as $\Lambda_{\gamma \gamma}\left(\boldsymbol{\pi}_{0}, \nu\right)_{\mathrm{DAV}}$, then we obtain

$$
\Lambda_{\gamma \gamma}\left(\boldsymbol{\pi}_{0}, \nu\right)_{\mathrm{DAV}}=Q(m, \nu) \times \Lambda\left(\boldsymbol{\pi}_{0}\right)_{\mathrm{MNL}},
$$

where

$$
Q(m, \nu)=\frac{m+\nu \sum_{x=2}^{m}\left(\frac{m-x}{x(m-1)}\left(\begin{array}{c}
m \\
x
\end{array}\right)\right)}{m+\nu \sum_{x=2}^{m}\left(\begin{array}{c}
m \\
x
\end{array}\right)} .
$$


This gives

$$
\Lambda\left(\boldsymbol{\pi}_{0}, \nu\right)_{\mathrm{DAV}}=\left[\begin{array}{cc}
Q(m, \nu) \times \Lambda\left(\boldsymbol{\pi}_{0}\right)_{\mathrm{MNL}} & 0 \\
0 & \Lambda_{\nu \nu}(m, \nu)
\end{array}\right] .
$$

Using the expression for $\Lambda\left(\pi_{0}, \nu\right)_{\mathrm{DAV}}$ and the $D$-optimality criterion we get the following result.

Theorem 2. For a set of $p$ contrasts of the entries in $\gamma$ and a constant but unknown $\nu$, the D-optimal design for the estimation of the contrasts of $\gamma$ over a set of competing designs $\mathfrak{X}$ when the MNL model is used will also be $D$-optimal for the estimation of the same contrasts and $\nu$ over the same set of competing designs when estimating the generalised Davidson ties model.

Proof. We begin by letting $B$ be the matrix of Equation 1. The contrasts in $B_{\gamma} \gamma$ are to be estimated in both models. Then the information matrix for the estimation of the contrasts in $B_{\gamma} \gamma$ as well as $\nu$ when the generalised Davidson model is used is

$$
C\left(\boldsymbol{\pi}_{0}, \nu\right)_{\mathrm{DAV}}=\left[\begin{array}{cc}
B_{\gamma} \Lambda_{\gamma \gamma}\left(\boldsymbol{\pi}_{0}, \nu\right) B_{\gamma}^{T} & \mathbf{0} \\
\mathbf{0} & \Lambda_{\nu \nu}(m, \nu)
\end{array}\right] .
$$

Substituting Equation 3 this into the expression for $C\left(\pi_{0}, \nu\right)_{\mathrm{DAV}}$, and simplifying, gives

$$
C\left(\boldsymbol{\pi}_{0}, \nu\right)_{\mathrm{DAV}}=\left[\begin{array}{cc}
Q(m, \nu) C\left(\boldsymbol{\pi}_{0}\right)_{\mathrm{MNL}} & 0 \\
0 & \Lambda_{\nu \nu}(m, \nu)
\end{array}\right] .
$$

Then

$$
\operatorname{det}\left(C\left(\boldsymbol{\pi}_{0}, \nu\right)_{\mathrm{DAV}}\right)=(Q(m, \nu))^{p} \times \Lambda_{\nu \nu}(m, \nu) \times \operatorname{det}\left(C\left(\boldsymbol{\pi}_{0}\right)_{\mathrm{MNL}}\right) .
$$

Since

$$
\operatorname{det}\left(\left(C\left(\boldsymbol{\pi}_{0}\right)_{\mathrm{MNL}}\right)_{\xi_{O P T}}\right) \geq \operatorname{det}\left(\left(C\left(\boldsymbol{\pi}_{0}\right)_{\mathrm{MNL}}\right)_{\xi}\right)
$$

for all $\xi \in \mathfrak{X}$, the relative efficiency of a generic design compared to the design $\xi_{\mathrm{OPT}}$, which is optimal for estimating the set of contrasts using the MNL model when the generalised Davidson ties model is used is

$$
\begin{aligned}
D_{\mathrm{eff}}\left(\xi, \xi_{\mathrm{OPT}}\right) & =\left(\frac{(Q(m, \nu))^{p} \times \Lambda_{\nu}(m, \nu) \times \operatorname{det}\left(C_{\xi, \mathrm{MNL}}\right)}{(Q(m, \nu))^{p} \times \Lambda_{\nu}(m, \nu) \times \operatorname{det}\left(C_{\xi_{\mathrm{OPT}, \mathrm{MNL}}}\right)}\right)^{\frac{1}{p+1}} \\
& =\left(\frac{\operatorname{det}\left(C_{\xi, \mathrm{MNL}}\right)}{\operatorname{det}\left(C_{\xi_{\mathrm{OPT}, \mathrm{MNL}}}\right)}\right)^{\frac{1}{p+1}} \\
& \leq 1,
\end{aligned}
$$


for all $\xi \in \mathfrak{X}$. Therefore, by the definition of $D$-optimality, the design $\xi_{\text {OPT }}$ is also optimal for estimating $\nu$ and the set of contrasts in $B_{\gamma} \gamma$ when using the generalised Davidson ties model.

\section{Conclusions}

One obvious generalisation would be to have a different ties parameter associated with subsets of different sizes. This would increase the number of $\nu$ from 1 to $m-1$ and

$$
B=\left[\begin{array}{cc}
B_{\gamma} & 0 \\
0 & I_{m-1}
\end{array}\right],
$$

but the same argument would establish that the MNL-optimal designs are still optimal in this case.

Another generalisation would be to consider different methods of incorporating ties into choice models. For example, we may wish to define a separate merit for finding each subset of the choice set equally attractive. We would then need to determine how well the MNL-optimal designs would perform using these models.

Burgess, L.B., and Street, D.J., 2003. Optimal designs for $2^{k}$ choice experiments. Communications in Statistics - Theory and Methods 32(11), 2185-2206.

Burgess, L.B., and Street, D.J., 2005. Optimal designs for choice experiments with asymmetric attributes. Journal of Statistical Planning and Inference 134(1), 288-301.

Davidson, R.R., 1970. On Extending the Bradley-Terry Model to Accommodate Ties in Paired Comparison Experiments. Journal of the American Statistical Association 65(319), 317-328.

El-Helbawy, A.T. and Ahmed, E.A. and Alharbey, A.H., 1994. Optimal Designs for Assymetrical Factorial Paired Comparison Experiments. Communications in Statistics - Simulation and Computation 22 (3), 663-681.

Gerard, K. and Sailsbury, C. and Street, D. and Pope, C. and Baxter, H., 2008. Is fast access to general practice all that should matter? A discrete choice experiment of patients' preferences. Journal of Health Services Research Policy 13 (2), 3-10. 
Louviere, J.J. Hensher, D.A. and Swait J.D., 2000. Stated Choice Methods. Cambridge University Press.

Luce, R.D., 1959. Individual Choice Behaviour: A Theoretical Analysis. John Wiley \& Sons, New York.

Street, D.J., and Burgess, L.B., 2004. Optimal and near-optimal pairs for the estimation of effects in 2-level choice experiments. Journal of Statistical Planning and Inference 118(1/2), 185-199.

Street, D.J., and Burgess, L., 2007. The Construction of Optimal Stated Choice Experiments. Wiley.

Train, K.E., 2002. Discrete Choice Methods with Simulation. Cambridge University Press. 